\title{
A meta-multiparametric framework: Application to the operation of bio-based energy supply chains
}

\author{
Sergio Medina, Ahmed Shokry, Javier Silvente and Antonio Espuña \\ Chemical Engineering Department, ETSEIB, Universitat Politècnica de Catalunya, 647 \\ Diagonal Avenue, 08028 Barcelona, Spain.
}

\begin{abstract}
This paper proposes a framework for the analysis of complex optimization problems under uncertainty. The usual multiparametric programing approach is skipped through the use of metamodels to mimic a set of already solved scenarios. Such framework has been applied to the operation of a bio-based energy supply chain, taking into account economic, environmental and social aspects: these objectives might be summarized in a single-objective formulation according to arbitrary economic criteria so, in order to assess the importance of the weighting factors used in the final decisions at different demand levels, a Kriging based metamodeling technique has been used to simplify the optimization procedures, leading to dramatic reductions in both the complexity of the methodology application and the required computational effort.
\end{abstract}

Keywords: metamodeling, kriging, uncertainty, multiparametric programming.

\section{Introduction}

The limited availability of fossil fuels and the concern related to the environmental impact associated to the use of these non-renewable resources have motivated the interest in the use of alternative technologies and biofuels for energy generation. One important practice is the use and exploitation of agroindustrial wastes (i.e. biomass) as a fuel for several power generation systems. The management of these situations generally faces to a challenging design and planning optimization problem which involves the capacities, cost and productions of the available energy generation units at each time instant (Silvente et al., 2013). Additionally, the network management is directly affected by several types of uncertainty in which we can highlight the demand uncertainty. Therefore, in order to ensure the reliability of the system, uncertainty must be considered due to the fact that the deterministic solution may become suboptimal or even infeasible (Kopanos and Pistikopoulos, 2013).

Different methods and tools have been proposed to consider uncertainty in the formulation of optimization models at different supply chain levels (demand, weather conditions, biomass supply, price variability across the time, etc). When this uncertainty affects not only the internal and external scenarios, but also the optimization parameters (like the importance of the different terms on the objective function), a way to analyze the effects of this uncertainty is the use of techniques referred as multiparametric programming. The main characteristic of multiparametric programming is its ability to obtain an optimal solution of the problem as a function of the uncertain/varying parameters and the region in the space of the parameters where these functions are valid (Sakizils et al., 2007). 
In this work, a methodology based on the metamodeling is proposed in order to create a multimetaparametric framework which provides practical advantages over existing methodologies in terms of simplicity and computational effort, leading to significant decision making information.

\section{Problem Statement}

The planning of a bio-based energy production supply chain to satisfy highest profit under uncertainty parameters and objective reduction is considered. The basic optimization problem formulation is based on a linear programming framework which considers the production capacity, storage levels, equipment capacity and sales per market as main decision variables. This model takes into account the production of energy through biomass considering the possibility of resizing the equipments of the existing energy plants.

A multiobjective evaluation of production levels to be managed and energy sales to the main power grid have been also included in order to maximize the profit taking care about environmental and social impacts as additional objectives. The mathematical model includes not only the mass and energy balances constraints associated to the energy production, but also the constraints required to describe the technologies involved (Dryer, chipper, Gasifier and transport units).

A kriging metamodeling technique (Shokry and Espuña, 2014) has been applied as a way to manage uncertain parameters based on its properties and potential of accurately approximating complex problems. The next terms describe the problem under study:

2.1. Inputs

Process data

- The set of materials $s \in S$, which includes raw, intermediates and final products.

- The tasks $i \in I$, which include on site treatments, pre-treatments and transportation.

- The set of economic criteria for environmental and social objectives.

- The set of locations (Fixed) $f c \in F C$, composed by suppliers (Biomass providers), intermediates (Pretreatment and treatment sites) and consumers (Markets).

- A time horizon $t \in T$, which represents the months of the year.

- A given expected energy demand profiles: for each short-term period and market, a different (uncertain) target value is considered.

Economic, Environmental and Social information

- $\quad$ Product and consumable prices are considered

- Environmental impacts for raw material production, process and transportation systems are also considered. The importance of this assessment on the decision making procedure is considered uncertain.

- The social impact is considered as a function of the size of the different installed processes, although again, The importance of this assessment on the decision making procedure is considered uncertain.

An optimization model based on the State Task Network formulation allows summarizing the information about the activities from all the SC nodes through a single variable set. The main decisions to be made to maximize the profit are related to: 
- Magnitude of the $\operatorname{task} P_{i, j, f c, f f c, t}$. This is the most important variable in the model and represents the magnitude of a task $i$ performed using technology $j$ during period $t$, whose origin is location $f c$ and destination is location $f f c$.

- Storage levels at each site and time.

- Size of installed equipment units.

The main equations of the mathematical formulation are now brief described. Storage levels of raw material in each site and at any time are specified in eq.1. Eq. 2 represents the energy balance in which the energy must respect the conservation law. the energy balance must be related to the amount of energy produced and consumed. Eq. 3 ensures that the production level and raw material processed in each facility will be greater or equal than a minimum utilization value defined by the decision maker and lower than the maximum capacity. Eq. 4 ensures that the raw material s purchased form site fc at time $t$ is lower or equal than an upper limit which at the same time is the physical availability of raw material. Eq. 5 is just a constraint that represents the fact that the demand could be satisfied partially for the energy produce using biomass. It is important to notice that even if the solution is treated as an uncertain parameter on the model it must be consider as a deterministic value since each change of demand value implies an isolated optimization procedure.

$$
\begin{array}{lr}
S_{s f t}-S_{s f t-1}=\sum_{f^{\prime}} \sum_{i \in T_{s}} \sum_{j \in\left(J_{i} \cap \widetilde{J}_{f^{\prime}}\right)} \alpha_{s i j} P_{i j f f^{\prime} t}-\sum_{f \prime} \sum_{i \in T_{s}} \sum_{j \in\left(J_{i} \cap \widetilde{J}_{f^{\prime}}\right)} \bar{\alpha}_{s i j} P_{i j f f \prime t} & \forall s, f, t \\
\sum_{s \in T_{s}} H V_{s} \alpha_{s i j} P_{i j f f^{\prime} t}=\sum_{s \in \bar{T}_{s}} H V_{s} \bar{\alpha}_{s i j} P_{i j f f^{\prime} t} & \forall i \in \bar{I}, j, f, t \\
\beta_{j f} F_{j f t-1} \leq \sum_{f^{\prime}} \sum_{i \in I_{j}} \theta_{i j f f^{\prime}} P_{i j f f^{\prime} t} \leq F_{j f t-1} & \forall f, j \in \tilde{J}_{f}, t \\
\sum_{f^{\prime}} \sum_{i \in \bar{T}_{s}} \sum_{j \in J_{i}} P_{i j f f^{\prime} t} \leq A_{s f t} & \forall s \in R M, f \in \text { Sup }, t
\end{array}
$$

The expected profit of the entire supply chain is calculated considering incomes (Esales) and costs. The costs include fixed cost $\left(F\right.$ Cost $\left._{t}\right)$ related at the investment and the variable cost (EPurch) including the transportation, acquisition and production cost in eq. 6 , subject to the previous constraints. Environmental impact is related to an LCA analysis. The main form of this analysis is in eq. 7. The social impact is calculated as the number of pretreatment and treatment sites installed as eq. 8 represents. In this equation the binary variable $V_{j f t}$ represents if a unit is installed or not. It is important to notice that in the proposed formulation the number of units installed will be the same for each of the parameters set since the superstructure is fixed, but it is considered in the model for comparison purposes.

$$
\text { Profit }_{t}=\text { Esales }_{t}-\left(\text { FCost }_{t}+\sum_{e} \text { EPurch }_{e t}\right)
$$




$$
\begin{aligned}
& \text { Impact }_{\text {overall }}^{2002}=\sum_{f} \sum_{g} \sum_{t} \sum_{a \in A_{g}} N_{\text {orm }} \zeta_{\text {ag }} I C_{\text {aft }} \\
& \forall g, f, t \\
& S o C=\sum_{j} \sum_{f} \sum_{t} V_{j f t} \\
& \forall j, f, t
\end{aligned}
$$

For more details of the model and symbology readers are suggested to read (Pérez-Fortes et al., 2012).

The original formulation is a multiobjective one which contemplates the profit, environmental and social impact as objectives. In the presented work the new objective function, which is the one to be maximized, is calculating transforming the objectives environmental and social impacts into an economic one applying an arbitrary criterion (WeightSoc and WeghtEnv). The objective function calculation is described by eq. 9 .

$$
O F=\sum_{t} \text { Profit }_{t}+(\text { SoC } * \text { WeightSoc })-\left(\text { Impact }_{\text {overall }}^{2002} * \text { WeightEnv }\right) \quad \forall t \quad \text { (9) }
$$

Notice that if the arbitrary criterion change the optimal solution could be suboptimal since this value affects directly the OF value. For this reason an kriging metamodel will be created in order to facilitate further optimization once the values of the arbitrary criterion changes. The same occurs with the Demand value that in this case is treated as an uncertain parameter.

\section{Methodology}

The proposed framework could be summarized in a set of steps that must be done in order to ensure its functionality.

1. Identification of the uncertain parameters to be analysed; confirmation of the corresponding upper and lower bounds.

2. Generation of a well distributed (representative) design of experiments.

3. Generation of the set of optimum solutions.

4. Training of the metamodel. In this particular case, the selected technique was "kriging", but any other suitable process may be used (support vector machines, ANNs, etc.). One metamodel for each one of the problem DOFs have to be trained.

5. Validation of the obtained metamodel. According to the results on this step, it may be considered to go back to point 3 and to generate a larger training set of experiments.

\section{Case study}

The supply chain introduced by Pérez-Fortes et al. (2012) has been used as case-study. It contemplates the bio-based energy production in a small district in Ghana. The 9 most important communities there were taken into account as suppliers, potential production sites and/or market sites for a 3 months planning horizon. The optimal fixed superstructure obtained for this particular case study (Pérez-Fortes et al., 2012) has been considered and, for this superstructure, the objective of this study includes the analysis on how the changes in the uncertain parameters (electricity demand and decision making criterion) affect the planning decissions. In the original work, these parameters were fixed to $50 € /$ Env_unit, $1000 € / \mathrm{Soc}$ _unit and $50000 \mathrm{kWh} / \mathrm{month}$ (WeightEnv, WeightSoc and 
Demand respectively); now these parameters are considered to vary in a range of 10-100 $€ /$ Env_unit, 100-10000€/Soc_unit, 49916-61009 kWh/month.

\subsection{Results and discussion}

Series of 25, 50, 75, 100, 125 and 150 experiments (combination of variables defining one single scenario) have been generated following Hammersly sampling technique and solved according to the proposed LP formulation using GAMS/CPLEX. Figure 1 shows the optimal objective values as a function of total demand and the environmental weight. The missing parameter under evaluation (Social weight) is not constant but even this it is worth noting that the response surface is clearly irregular, confirming that the parametric function may be nonlinear although the basic problem formulation is linear.

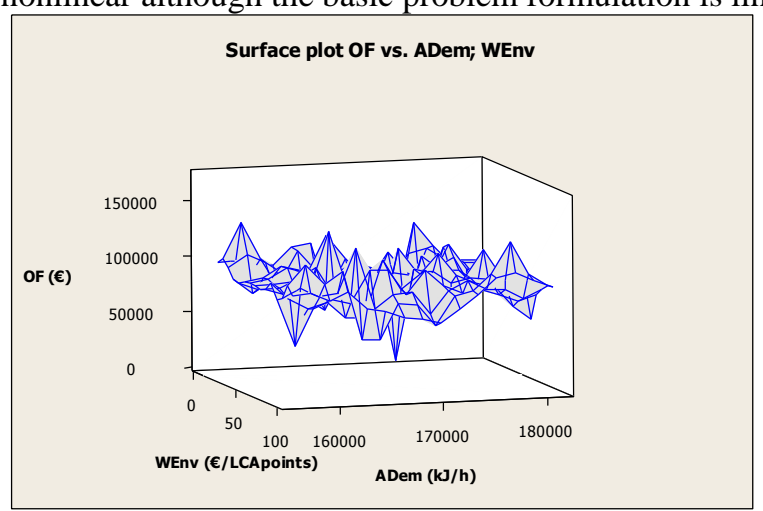

Figure 1. Behavior of the optimal objective function for different values in weightEnv and total demand parameters.

Figure 2 shows the kriging model cross-validation using sets of experiments of different sizes, and exhibits (a) the correlation between the estimated Objective Function obtained by a surrogate model trained using a reduced number of experiments, and the corresponding real optimum values (optimization of the original model), and (b) the quality of the metamodel as a function of the size of the training set. Although an obvious improvement in the quality of the fitting with the size of the training set is observed, the fitting is good enough to justify the use of the surrogate model, even in cases where the size of the training set is quite small. Similar plots are obtaining for the models fitted to reproduce the resulting values of the DOFs at their optimum values. 

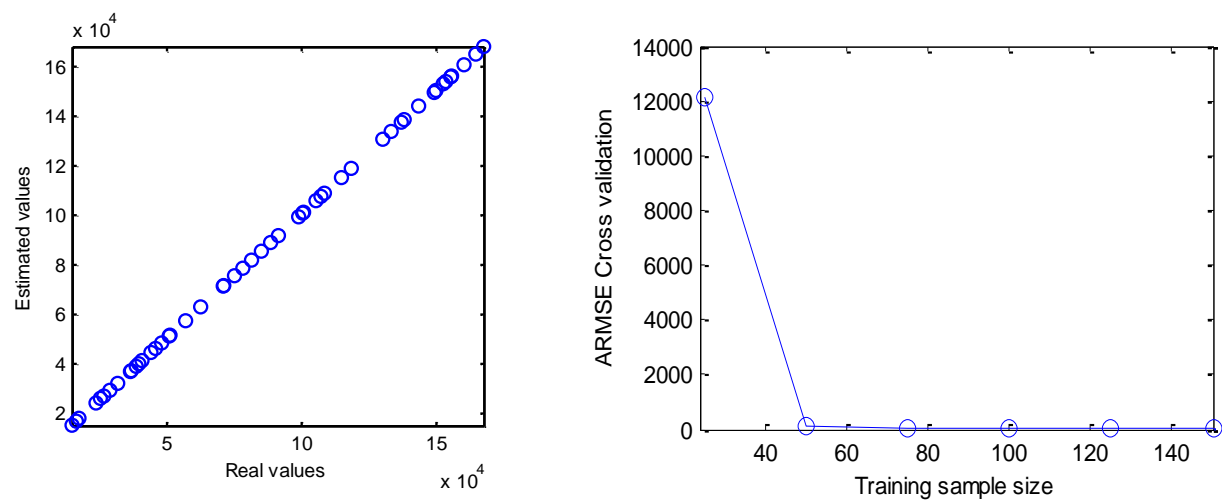

Figure 2. Cross-validation a) for a set of 50 experiments; b) as a function of the size of the training set.

Finally, Table 1 illustrates the reduction in the optimization time with the use of the metamodel. Although the solution of the 150 experiments requires a relatively high computational effort (this problem may become very important when a more complicated model is to be solved), once the surrogate model has been created the optimization time drops dramatically: for this example, the time to obtain the solution is reduced in more than 3 orders of magnitude $(1 / 4,895)$, and of course higher reductions would be obtained for a more complex optimization problem.

Table 1. Computational effort required.

\begin{tabular}{ccc}
\hline & $\begin{array}{c}\text { Math. Programming } \\
\text { (GAMS/CEPPLEX) }\end{array}$ & Kriging metamodel \\
\hline $\begin{array}{c}\text { Training effort (CPU s) } \\
\text { Optimization effort (CPU } \\
\text { s) }\end{array}$ & (model building) & $\begin{array}{c}\text { (model building }+ \\
3300 \mathrm{~s}(150 \text { experiments) + } \\
70 \mathrm{~s} \text { (training)) }\end{array}$ \\
\hline
\end{tabular}

(*)HP-dc7900, Intel core 2-duo 3, 16 GHz, RAM 6GB.

\section{Conclusions}

In order to solve optimization problems under uncertainty, which usually requires programming, can be also successfully solved employing a framework based on Kriging metamodel. The resulting model behaves like a useful metamultiparametric framework. This has been proved in relatively simple case studies. More work is required to develop a robust framework to handle bigger and more complex problems (Highly nonlinear models, MIP problems, etc.).

\section{Acknowledgements}

The authors would like to thank the financial support received from the Spanish "Ministerio de Economía y Competitividad" and the European Regional Development Fund (both funding the research Project SIGERA, DPI2012-37154-C02-01) and from the 
"Ministerio de Economía y Competitividad" (FPI, BES-2010-036099), from the Generalitat de Catalunya (2014-SGR-1092-CEPEiMA) and the Mexican National Council for Science and Technology (CONACyT).

\section{References}

G.M. Kopanos, E.N. Pistikopoulos, 2013, Ind. \& Eng. Chem. Res., 53, 4366-4386

M. Pérez-Fortes, J.M. Laínez, P. Arranz-Piera, E. Velo, L. Puigjaner, 2012, Energy, 44, 79-95

A. Shokry, A. Espuña, 2014, Applying metamodels and sequential sampling for constrained optimization of process operations, Lecture Notes in Computer Science, 396-407.

V. Sakizils, K.I. Kouramas, E.N. Pistikopoulous, 2007, Linear Model Predictive Control via Multiparametric programming. Multi-Parametric model-Based Control, volume 2, 275 pages.

J. Silvente, A. Aguirre, G. Crexells, M. Zamarripa, C. Méndez, M. Graells, A. Espuña, 2013, Computer-Aided Chemical Engineering, 32, 553-558

Z. Li, M.G. Ierapetritou M.G, 2008, AIChE Journal, 54, 2610-2623.

Pèrez-Fontes M. Conceptual design of alternative energy systems from biomass, PhD. Thesis, UPC, Barcelona, Spain. (2011) 